\title{
On maximum modulus for the derivative of a polynomial
}

\begin{abstract}
If $P(z)$ is a polynomial of degree $n$, having all its zeros in the disk $|z| \leq k, k \geq 1$, then it was shown by Govil [Proc. Amer. Math. Soc. 41, no. 2 (1973), 543-546] that

$$
\max _{|z|=1}\left|P^{\prime}(z)\right| \geq \frac{n}{1+k^{n}} \max _{|z|=1}|P(z)| .
$$

In this paper, we obtain generalization as well as improvement of above inequality for the polynomial of the type $P(z)=c_{0}+\sum_{\nu=\mu}^{n} c_{\nu} z^{\nu}, 1 \leq \mu \leq n$. Also we generalize a result due to Dewan and Mir [Southeast Asian Bull. Math. 31 (2007), 691-695] in this direction.
\end{abstract}

1. Introduction and statement of results. If $P(z)$ is a polynomial of degree $n$ and $P^{\prime}(z)$ its derivative, then according to a famous result known as Bernstein's inequality (for reference see [1]), we have

$$
\max _{|z|=1}\left|P^{\prime}(z)\right| \leq n \max _{|z|=1}|P(z)| \text {. }
$$

For the polynomial $P(z)$, it is well known as a simple consequence of maximum modulus principle (for reference see [7, p. 158, problem 269]) that for $R \geq 1$,

$$
\max _{|z|=R}|P(z)| \leq R^{n} \max _{|z|=1}|P(z)| .
$$

2000 Mathematics Subject Classification. 30A10, 30C10, 30C15.

Key words and phrases. Polynomials, inequalities, derivatives, zeros.

The work is supported by Council of Scientific and Industrial Research, New Delhi, under grant F. No. 9/466(95)/2007-EMR-I. 
Both the inequalities (1.1) and (1.2) are sharp and equality holds for $P(z)=$ $\alpha z^{n}$, where $|\alpha|=1$.

Turán [9] considered that if $P(z)$ is a polynomial of degree $n$, having all its zeros in $|z| \leq 1$, then

$$
\max _{|z|=1}\left|P^{\prime}(z)\right| \geq \frac{n}{2} \max _{|z|=1}|P(z)| .
$$

The result is best possible and equality holds for $P(z)=\alpha+\beta z^{n}$, where $|\alpha|=|\beta|$.

As a generalization of inequality (1.3), Govil [3] proved the following result.

Theorem A. If $P(z)$ is a polynomial of degree $n$, having all its zeros in the disk $|z| \leq k, k \geq 1$, then

$$
\max _{|z|=1}\left|P^{\prime}(z)\right| \geq \frac{n}{1+k^{n}} \max _{|z|=1}|P(z)| .
$$

The result is best possible and equality holds for $P(z)=\left(z^{n}+k^{n}\right)$.

For the polynomial not vanishing in $|z|<k, k \leq 1$, Govil [4] proved that if $P(z)$ has all its zeros on $|z|=k, k \leq 1$, then

$$
\max _{|z|=1}\left|P^{\prime}(z)\right| \leq \frac{n}{k^{n}+k^{n-1}} \max _{|z|=1}|P(z)| .
$$

While seeking for the better bound of the inequality (1.5), recently Dewan and Mir [2] proved the following result under the same hypothesis.

Theorem B. If $P(z)=\sum_{\nu=0}^{n} c_{\nu} z^{\nu}$ is a polynomial of degree $n$, having all its zeros on $|z|=k, k \leq 1$, then

$$
\max _{|z|=1}\left|P^{\prime}(z)\right| \leq \frac{n}{k^{n}}\left\{\frac{n\left|c_{n}\right| k^{2}+\left|c_{n-1}\right|}{n\left|c_{n}\right|\left(1+k^{2}\right)+2\left|c_{n-1}\right|}\right\} \max _{|z|=1}|P(z)| .
$$

In this paper, we consider a class of polynomials $P(z)=c_{0}+\sum_{\nu=\mu}^{n} c_{\nu} z^{\nu}$, $1 \leq \mu \leq n$ and generalize as well as improve upon Theorem $\mathrm{A}$ and also generalize Theorem B by proving the following results.

Theorem 1. If $P(z)=c_{0}+\sum_{\nu=\mu}^{n} c_{\nu} z^{\nu}, 1 \leq \mu<n$ is a polynomial of degree $n$, having all its zeros in the disk $|z| \leq k, k \geq 1$, then

$$
\max _{|z|=1}\left|P^{\prime}(z)\right| \geq \frac{n}{1+k^{n-\mu+1}} \max _{|z|=1}|P(z)| .
$$

The result is best possible and equality holds for

$$
P(z)=\left(z^{n-\mu+1}+k^{n-\mu+1}\right)^{\frac{n}{n-\mu+1}} .
$$

Remark 1. If we take $\mu=1$ in Theorem 1, then inequality (1.7) reduces to inequality (1.4) due to Govil [3]. 
Theorem 2. If $P(z)=c_{n} z^{n}+\sum_{\nu=\mu}^{n} c_{n-\nu} z^{n-\nu}, 1 \leq \mu<n$ is a polynomial of degree $n$, having all its zeros on $|z|=k, k \leq 1$, then

$$
\begin{aligned}
\max _{|z|=1} \mid & P^{\prime}(z) \mid \\
& \leq \frac{n}{k^{n-\mu+1}}\left(\frac{n\left|c_{n}\right| k^{2 \mu}+\mu\left|c_{n-\mu}\right| k^{\mu-1}}{\mu\left|c_{n-\mu}\right|\left(1+k^{\mu-1}\right)+n\left|c_{n}\right| k^{\mu-1}\left(1+k^{\mu+1}\right)}\right) \max _{|z|=1}|P(z)| .
\end{aligned}
$$

Remark 2. If we take $\mu=1$ in Theorem 2 , then the above inequality reduces to the inequality (1.6) due to Dewan and Mir [2].

Theorem 3. If $P(z)=c_{0}+\sum_{\nu=\mu}^{n} c_{\nu} z^{\nu}, 1 \leq \mu<n$ is a polynomial of degree $n$, having all its zeros in the disk $|z| \leq k, k \geq 1$, then

$$
\max _{|z|=1}\left|P^{\prime}(z)\right| \geq \frac{n}{1+k^{n-\mu+1}}\left\{\max _{|z|=1}|P(z)|+\min _{|z|=k}|P(z)|\right\} .
$$

The result is best possible and equality holds for

$$
P(z)=\left(z^{n-\mu+1}+k^{n-\mu+1}\right)^{\frac{n}{n-\mu+1}} .
$$

If we choose $\mu=1$ in Theorem 3, then inequality (1.8) reduces to following result due to Govil [5].

Corollary 1. If $P(z)$ is a polynomial of degree $n$, having all its zeros in the disk $|z| \leq k, k \geq 1$, then

$$
\max _{|z|=1}\left|P^{\prime}(z)\right| \geq \frac{n}{1+k^{n}}\left\{\max _{|z|=1}|P(z)|+\min _{|z|=k}|P(z)|\right\} .
$$

The result is best possible and equality holds for $P(z)=z^{n}+k^{n}$.

2. Lemmas. We need the following lemmas for the proofs of these theorems.

Lemma 1. If $P(z)=c_{0}+\sum_{\nu=\mu}^{n} c_{\nu} z^{\nu}, 1 \leq \mu<n$ is a polynomial of degree $n$, having all its zeros in the disk $|z| \leq k, k \geq 1$, then for $|z|=1$

$$
k^{n+\mu-3}\left|Q^{\prime}(z)\right| \leq\left|P^{\prime}\left(k^{2} z\right)\right|,
$$

where $Q(z)=z^{n} \overline{P(1 / \bar{z})}$.

Proof of Lemma 1. Since the polynomial $P(z)$ has all its zeros in $|z| \leq k$, $k \geq 1$, therefore the polynomial $F(z)=P(k z)$ has all its zeros in the unit disk $|z| \leq 1$. Now if $G(z) \equiv z^{n} \overline{F(1 / \bar{z})} \equiv z^{n} \overline{P(k / \bar{z})} \equiv k^{n} Q(z / k)$, then all the zeros of $G(z)$ lie in $|z| \geq 1$. Since $|F(z)|=|G(z)|$ on $|z|=1$, it follows by maximum modulus principle that $|G(z)| \leq|F(z)|$ on $|z| \geq 1$. Hence for every complex number $\lambda$ with $|\lambda|>1$, it follows by Rouche's theorem that the polynomial $G(z)-\lambda F(z)$ has all its zeros in $|z|<1$. By Gauss-Lucas theorem the polynomial $G^{\prime}(z)-\lambda F^{\prime}(z)$ has all its zeros in $|z|<1$, which implies

$$
\left|G^{\prime}(z)\right| \leq\left|F^{\prime}(z)\right| \text { for }|z| \geq 1
$$


Substituting for $F(z)$ and $G(z)$ in (2.2), we get

$$
k^{n-1}\left|Q^{\prime}(z / k)\right| \leq k\left|P^{\prime}(k z)\right| \text { for }|z| \geq 1 .
$$

Since $c_{1}=c_{2}=\cdots=c_{\mu-1}=0$, from (2.3), we get

$$
k^{n-1}\left|Q^{\prime}(z / k)\right| \leq k^{\mu}\left|\sum_{\nu=\mu}^{n} \nu c_{\nu}(k z)^{\nu-\mu}\right| \text { for }|z| \geq 1 .
$$

In fact (2.4) holds for $|z|=1$. But $\sum_{\nu=\mu}^{n} \nu c_{\nu}(k z)^{\nu-\mu} \neq 0$ in $|z|>1$, by maximum modulus principle it also holds for $|z|>1$. Taking $k z$ instead of $z$ in (2.4), we have

$$
k^{n-1}\left|Q^{\prime}(z)\right| \leq k^{\mu}\left|\sum_{\nu=\mu}^{n} \nu c_{\nu}\left(k^{2} z\right)^{\nu-\mu}\right| \quad \text { for }|z| \geq 1 / k .
$$

In particular,

$$
k^{n-1}\left|Q^{\prime}(z)\right| \leq k^{\mu}\left|\sum_{\nu=\mu}^{n} \nu c_{\nu}\left(k^{2} z\right)^{\nu-\mu}\right| \text { for }|z|=1,
$$

this implies

$$
k^{n-1}\left|Q^{\prime}(z)\right| \leq k^{2-\mu}\left|\sum_{\nu=\mu}^{n} \nu c_{\nu}\left(k^{2} z\right)^{\nu-1}\right| \quad \text { for }|z|=1 .
$$

Consequently

$$
k^{n+\mu-3}\left|Q^{\prime}(z)\right| \leq\left|P^{\prime}\left(k^{2} z\right)\right| \text { for }|z|=1 .
$$

This completes the proof of Lemma 1.

Lemma 2. If $P(z)=c_{0}+\sum_{\nu=\mu}^{n} c_{\nu} z^{\nu}, 1 \leq \mu<n$ is a polynomial of degree $n$, having all its zeros in the disk $|z| \leq k, k \geq 1$, then

$$
\max _{|z|=1}\left|Q^{\prime}(z)\right| \leq k^{n-\mu+1} \max _{|z|=1}\left|P^{\prime}(z)\right|,
$$

where $Q(z)=z^{n} \overline{P(1 / \bar{z})}$.

Proof of Lemma 2. By Lemma 1, we have

$$
\max _{|z|=1}\left|Q^{\prime}(z)\right| \leq \frac{1}{k^{n+\mu-3}} \max _{|z|=k^{2}}\left|P^{\prime}(z)\right| .
$$

Using inequality (1.2) for the polynomial $P^{\prime}(z)$ with $R=k^{2} \geq 1$, we have

$$
\max _{|z|=k^{2}}\left|P^{\prime}(z)\right| \leq k^{2 n-2} \max _{|z|=1}\left|P^{\prime}(z)\right| .
$$

Combining this with (2.5), the lemma follows. 
Lemma 3. If $P(z)=c_{n} z^{n}+\sum_{\nu=\mu}^{n} c_{n-\nu} z^{n-\nu}, 1 \leq \mu<n$ is a polynomial of degree $n$, having no zero in the disk $|z|<k, k \leq 1$, then

$$
k^{n-\mu+1} \max _{|z|=1}\left|P^{\prime}(z)\right| \leq \max _{|z|=1}\left|Q^{\prime}(z)\right|,
$$

where $Q(z)=z^{n} \overline{P(1 / \bar{z})}$.

Proof of Lemma 3. If $P(z)$ has no zero in $|z|<k, k \leq 1$, then $Q(z)=$ $z^{n} \overline{P(1 / \bar{z})}$ has all its zeros in $|z| \leq 1 / k, 1 / k \geq 1$. Thus applying Lemma 2 to the polynomial $Q(z)$, we get

$$
\max _{|z|=1}\left|P^{\prime}(z)\right| \leq \frac{1}{k^{n-\mu+1}} \max _{|z|=1}\left|Q^{\prime}(z)\right|,
$$

and the lemma follows.

Lemma 4. If $P(z)$ is a polynomial of degree $n$, then for $|z|=1$

$$
\left|P^{\prime}(z)\right|+\left|Q^{\prime}(z)\right| \leq n \max _{|z|=1}|P(z)|
$$

where $Q(z)=z^{n} \overline{P(1 / \bar{z})}$.

The above lemma is a special case of a result due to Govil and Rahman [6].

Lemma 5. If $P(z)=c_{0}+\sum_{\nu=\mu}^{n} c_{\nu} z^{\nu}, 1 \leq \mu \leq n$ is a polynomial of degree $n$, having no zero in the disk $|z|<k, k \geq 1$, then for $|z|=1$

$$
k^{\mu+1}\left\{\frac{\mu\left|c_{\mu}\right| k^{\mu-1}+n\left|c_{0}\right|}{n\left|c_{0}\right|+\mu\left|c_{\mu}\right| k^{\mu+1}}\right\}\left|P^{\prime}(z)\right| \leq\left|Q^{\prime}(z)\right|
$$

and

where $Q(z)=z^{n} \overline{P(1 / \bar{z})}$.

$$
\frac{\mu}{n}\left|\frac{c_{\mu}}{c_{0}}\right| k^{\mu} \leq 1
$$

The above lemma was given by Qazi [8, Remark and proof of Lemma 1].

Lemma 6. If $P(z)=c_{n} z^{n}+\sum_{\nu=\mu}^{n} c_{n-\nu} z^{n-\nu}, 1 \leq \mu \leq n$ is a polynomial of degree $n$, having all its zeros on $|z|=k, k \leq 1$, then for $|z|=1$

$$
k^{\mu-1}\left\{\frac{n\left|c_{n}\right| k^{\mu+1}+\mu\left|c_{n-\mu}\right|}{\mu\left|c_{n-\mu}\right|+n\left|c_{n}\right| k^{\mu-1}}\right\}\left|P^{\prime}(z)\right| \geq\left|Q^{\prime}(z)\right|
$$

and

$$
\frac{\mu}{n}\left|\frac{c_{n-\mu}}{c_{n}}\right| \leq k^{\mu}
$$

where $Q(z)=z^{n} \overline{P(1 / \bar{z})}$.

Proof of Lemma 6. Since $P(z)$ has all its zeros on $|z|=k, k \leq 1$, therefore $Q(z)=z^{n} \overline{P(1 / \bar{z})}$ has all its zeros on $|z|=1 / k, 1 / k \geq 1$. Now applying Lemma 5 to polynomial $Q(z)$ and result follows. 
The following lemma is due to Govil [3].

Lemma 7. If $P(z)$ is a polynomial of degree $n$ and $P(z) \equiv Q(z)$, then for $|z|=1$

$$
\max _{|z|=1}\left|P^{\prime}(z)\right|=\frac{n}{2} \max _{|z|=1}|P(z)|,
$$

where $Q(z)=z^{n} \overline{P(1 / \bar{z})}$.

\section{Proofs of the theorems.}

Proof of Theorem 1. For every $\epsilon$ with $|\epsilon|=1$, the polynomial $P^{*}(z)=$ $\frac{1}{2}(P(z)+\epsilon Q(z))$ satisfies $P^{*}(z) \equiv z^{n} \overline{P^{*}(1 / \bar{z})}$, hence by Lemma 7 , we have

$$
\max _{|z|=1}\left|P^{\prime}(z)+\epsilon Q^{\prime}(z)\right|=\frac{n}{2} \max _{|z|=1}|P(z)+\epsilon Q(z)| .
$$

This implies

$$
\max _{|z|=1}\left|P^{\prime}(z)\right|+\max _{|z|=1}\left|Q^{\prime}(z)\right| \geq \frac{n}{2} \max _{|z|=1}|P(z)+\epsilon Q(z)| .
$$

Choosing the argument of $\epsilon$ on right hand side, we get

$$
\max _{|z|=1}\left|P^{\prime}(z)\right|+\max _{|z|=1}\left|Q^{\prime}(z)\right| \geq n \max _{|z|=1}|P(z)| .
$$

Which further on applying Lemma 2, gives

$$
\max _{|z|=1}\left|P^{\prime}(z)\right|+k^{n-\mu+1} \max _{|z|=1}\left|P^{\prime}(z)\right| \geq n \max _{|z|=1}|P(z)|
$$

and the theorem follows.

Proof of Theorem 2. Let $z_{0}$ be a point on $|z|=1$, such that $\left|Q^{\prime}\left(z_{0}\right)\right|=$ $\max _{|z|=1}\left|Q^{\prime}(z)\right|$, then by Lemma 4 , it follows that

$$
\left|P^{\prime}\left(z_{0}\right)\right|+\max _{|z|=1}\left|Q^{\prime}(z)\right| \leq n \max _{|z|=1}|P(z)| .
$$

Combining inequality (3.1) with Lemma 6, we get

$$
\frac{1}{k^{\mu-1}}\left(\frac{\mu\left|c_{n-\mu}\right|+n\left|c_{n}\right| k^{\mu-1}}{n\left|c_{n}\right| k^{\mu+1}+\mu\left|c_{n-\mu}\right|}\right)\left|Q^{\prime}\left(z_{0}\right)\right|+\max _{|z|=1}\left|Q^{\prime}(z)\right| \leq n \max _{|z|=1}|P(z)|,
$$

which implies

$$
\begin{aligned}
& \left(\frac{\mu\left|c_{n-\mu}\right|\left(1+k^{\mu-1}\right)+n\left|c_{n}\right| k^{\mu-1}\left(1+k^{\mu+1}\right)}{n\left|c_{n}\right| k^{2 \mu}+\mu\left|c_{n-\mu}\right| k^{\mu-1}}\right) \max _{|z|=1}\left|Q^{\prime}(z)\right| \\
& \leq n \max _{|z|=1}|P(z)| .
\end{aligned}
$$

Inequality (3.2), when combined with Lemma 3, gives

$$
\begin{aligned}
& k^{n-\mu+1}\left(\frac{\mu\left|c_{n-\mu}\right|\left(1+k^{\mu-1}\right)+n\left|c_{n}\right| k^{\mu-1}\left(1+k^{\mu+1}\right)}{n\left|c_{n}\right| k^{2 \mu}+\mu\left|c_{n-\mu}\right| k^{\mu-1}}\right) \max _{|z|=1}\left|P^{\prime}(z)\right| \\
& \leq n \max _{|z|=1}|P(z)| .
\end{aligned}
$$


This implies

$$
\begin{aligned}
\max _{|z|=1} & \left|P^{\prime}(z)\right| \\
& \leq \frac{n}{k^{n-\mu+1}}\left(\frac{n\left|c_{n}\right| k^{2 \mu}+\mu\left|c_{n-\mu}\right| k^{\mu-1}}{\mu\left|c_{n-\mu}\right|\left(1+k^{\mu-1}\right)+n\left|c_{n}\right| k^{\mu-1}\left(1+k^{\mu+1}\right)}\right) \max _{|z|=1}|P(z)|,
\end{aligned}
$$

which completes the proof of Theorem 2 .

Proof of Theorem 3. If $m=\min _{|z|=k}|P(z)|$, then for every $\alpha$ with $|\alpha|<$ 1 , the polynomial $P(z)+\alpha m$ has all its zeros in $|z| \leq k, k \geq 1$. This is clear if $P(z)$ has a zero on $|z|=k$, because in that case $m=0$ and therefore $P(z)+\alpha m=P(z)$. In case $P(z)$ has no zero on $|z|=k$, then for every $\alpha$ with $|\alpha|<1$, we have $|P(z)|>m|\alpha|$ on $|z|=k$ and on applying Rouche's theorem the result will follow. Thus $P(z)+\alpha m$ has all its zeros in $|z| \leq k$, $k \geq 1$ and hence, applying Theorem 1 to $P(z)+\alpha m$, we get

$$
\max _{|z|=1}\left|P^{\prime}(z)\right| \geq \frac{n}{1+k^{n-\mu+1}} \max _{|z|=1}|P(z)+\alpha m| .
$$

Now choosing argument of $\alpha$ on the right hand side and letting $|\alpha| \rightarrow 1$, we get

$$
\max _{|z|=1}\left|P^{\prime}(z)\right| \geq \frac{n}{1+k^{n-\mu+1}}\left\{\max _{|z|=1}|P(z)|+\min _{|z|=k}|P(z)|\right\} .
$$

This completes the proof of Theorem 3 .

Remark 3. For $\mu=n$ Theorems 1,2 and 3 hold if polynomial satisfies the condition $\left|c_{0}\right| \leq k\left|c_{n}\right|$.

\section{REFERENCES}

[1] Bernstein, S., Lecons sur les propriétés extrémales et la meilleure approximation desfonctions analytiques d'une variable réelle, Gauthier Villars, Paris, 1926.

[2] Dewan, K. K., Mir, A., Note on a theorem of S. Bernstein, Southeast Asian Bull. Math. 31 (2007), 691-695.

[3] Govil, N. K., On the derivatives of a polynomial, Proc. Amer. Math. Soc. 41, no. 2 (1973), 543-546.

[4] Govil, N. K., On a theorem of S. Bernstein, J. Math. Phys. Sci., 14, no. 2 (1980), 183-187.

[5] Govil, N. K., Some inequalities for derivatives of polynomials, J. Approx. Theory 66 (1991), 29-35.

[6] Govil, N. K., Rahman, Q. I., Functions of exponential type not vanishing in a half plane and related polynomials, Trans. Amer. Math. Soc. 137 (1969), 501-517.

[7] Pólya, G., Szegö, G., Problems and Theorems in Analysis, Vol. 1, Springer-Verlag, New York, 1972.

[8] Qazi, M. A., On the maximum modulus of polynomials, Proc. Amer. Math. Soc. 115 (1992), 337-343.

[9] Turán, P., Über die ableitung von Polynomen, Compositio Math. 7 (1939), 89-95. 
K. K. Dewan

Department of Mathematics

Faculty of Natural Sciences

Jamia Millia Islamia

(Central University)

New Delhi-110025

India

e-mail: kkdewan1230yahoo.co.in
Sunil Hans

Department of Mathematics

Faculty of Natural Sciences

Jamia Millia Islamia

(Central University)

New Delhi-110025

India

e-mail: sunil.hans82@yahoo.com

Received February 12, 2009 